\title{
Soportes neurales de las emociones: un acercamiento descriptivo
}

\section{Neural supports of emotions: a descriptive approach}

DOI: $10.46932 / \operatorname{sfjdv2n4-045~}$

Received in: March 1st, 2021

Accepted in: May 30th, 2021

\author{
Franyelit Suárez-Carreño \\ Facultad de Ingeniería y Ciencias Aplicadas, Carrera de Ingeniería Industrial \\ Universidad de las Américas- Quito Ecuador \\ E-mail: Franyelit.suarez@udla.edu.ec
}

\begin{abstract}
RESUMEN
En este trabajo se presenta una revisión de las emociones desde los procesos cognoscitivos cerebrales, tomando en cuenta algunos estudios neurobiológicos experimentales. Se analizan los sistemas sensoriales, las reacciones fisiológicas del sistema nervioso central, y se evalúan los estímulos emocionales. Se evidencia que las expresiones subjetivas y las reacciones físicas de las emociones están condicionadas a los patrones sociales, costumbres familiares y principalmente a estereotipos morales de las personas. Entre las características observadas prevalecen las emociones auto sancionarías y las de empatía con el otro. Finalmente fue posible reconocer que la restricción de las emociones en las personas puede ocasionar conductas psicológicas que perjudican su relación con el entorno. Además, se producen conductas antisociales, falta de comprensión de los sentimientos ajenos, incumplimiento de las reglas, entre otras actividades y estándares sociales.
\end{abstract}

Palabras clave: emociones humanas, estímulos emocionales, neurobiología de las emociones.

\begin{abstract}
This work presents a review of emotions from the cognitive processes of the brain, taking into account some experimental neurobiological studies. Sensory systems are analyzed, physiological reactions of the central nervous system, and emotional stimuli are evaluated. It is evidenced that subjective expressions and physical reactions of emotions are conditioned to social patterns, family customs and mainly to moral stereotypes of people. Among the observed characteristics, self-sanctioning emotions and those of empathy with the other prevail. Finally, it was possible to recognize that the restriction of emotions in people can cause psychological behaviors that damage their relationship with the environment. In addition, antisocial behaviors occur, lack of understanding of the feelings of others, breach of rules, among other activities and social standard.
\end{abstract}

Keywords: human emotions, emotional stimuli, neurobiology of emotions.

\section{INTRODUCCIÓN}

En el año 1872, Charles Darwin (Fernández-Berrocal, 2009) publicó los resultados de uno de los trabajos más controversiales de los últimos cien años, en el que expuso los resultados de la recopilación de información de sus treinta años de estudios sobre las emociones. En estos resultados Darwin manifestó dos ideas fundamentales; las emociones son innatas y universales, y que las emociones son producto de 
la evolución. Darwin postuló que las expresiones faciales son un reflejo de las emociones y que estas no son exclusivas de los humanos (Ramírez, 2001). Sin embargo, (Harlow \& Harlow, 1962), (Reite \& Short, 1981) afirman que la sonrisa y el llanto son exclusivas de los humanos, como manifestación de las emociones como mecanismo de adaptación de la especie (Montagu, 1959).

Las emociones pueden generar diferentes respuestas en el organismo (Palacios, 2017), tanto favorables como adversas, entre los que se cuentan la alegría, tristeza, enfado, desagrado, entre otros. Estas emociones están sujetas a las condiciones sociales y culturales, que afectan la conducta de las personas y por ende, influyen en la percepción de la realidad que desencadena dichas emociones (Palacios, 2017), (Surrallés, 2005), (Bourdin, 2016).

Tomando en cuenta lo expuesto en (Ibañez, 2009) y (Wierzbicka, 1986) se establecen las emociones básicas, que a partir de sus compuestos dan origen a emociones secundarias, así entonces se lista el interés, la alegría, la sorpresa, la tristeza, la ira, la repugnancia, el desprecio o desdén, el miedo o temor, la vergüenza o la timidez y la culpa. De modo que una emoción secundaria podría ser por ejemplo el espanto, producto del miedo con la sorpresa.

Las emociones que alcanzan niveles intensos y se presentan con mucha frecuencia tienden a producir cambios en la conducta, de manera que se dejan a un lado los aspectos saludables y se empiezan a desarrollar conductas adictivas que afectan el estado de salud, desencadenando reacciones fisiológicas que determinan el estrés (Moure, 2011).

El estrés subyace en las emociones, por cuanto sus efectos inciden tanto en la salud física y mental como en el rendimiento laboral y desempeño social de las personas (Naranjo, 2009). El problema del estrés está asociado a los requerimientos de la modernidad, centrada en la búsqueda de alternativas para una mejor calidad de vida, y por ende no se evalúan las consecuencias de la salud física y mental de las personas afectadas (Naranjo, 2009), (Martínez \& Díaz, 2007).

En este trabajo se describen los soportes neurales de las emociones, desde los fundamentos biológicos hasta su impacto en la utilización de las redes neuronales artificiales. Se plantea el algoritmo HOG para la caracterización del espectro de voz y se contrasta con la metodología de caracterización de fisionomía facial, validadas entonces con 280 datos extraídos de los estudiantes de la carrera de ingeniería industrial de la Universidad de las Américas del Ecuador.

El trabajo está organizado en cinco secciones, en la segunda se expone la metodología que hace posible la elaboración de pruebas de funcionamiento y validación, en la tercera se describen los resultados, en la cuarta sección se hace un análisis de los resultados y finalmente se exponen las conclusiones y recomendaciones. 


\section{METODOLOGÍA}

Para el desarrollo de este trabajo se ha considerado la evaluación teórica de las emociones a través del espectro de voz, que según (Palacios, 2017) ofrece una característica única en los humanos para determinar sus estados emocionales. Algunos autores (Ramírez, 2001) aseguran que la voz humana no puede obviar las condiciones emocionales que posee cada individuo. Además, se ha podido demostrar que el espectro de voz posee vibraciones únicas entre una persona y otra, y por tanto no puede ser confundible.

La figura 1 muestra un diagrama de los procesos ejecutados para determinar el posible estado emocional de una persona a partir de la frecuencia de voz. En ella se observa que el sistema fue analizado por un algoritmo neuronal que vincula las características vocales y las normaliza para lograr una mejor precisión del sonido.

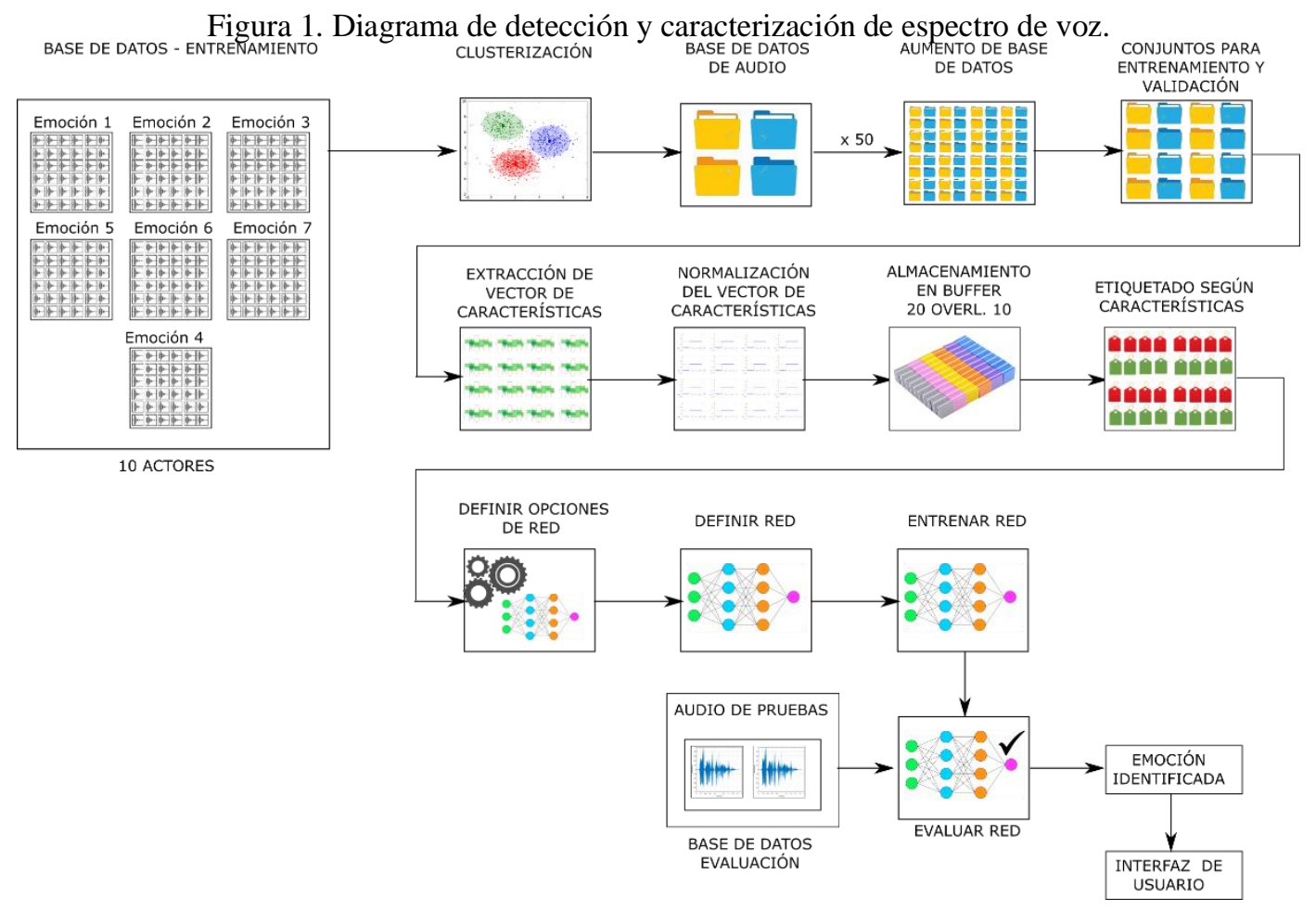

Además, para el proceso planteado en este trabajo se ha comparado el resultado de la validación de voz con el obtenido en la medición de fisionomía del rostro para detección de emociones. En ella se han evaluado las siete emociones básicas dadas por ira, asco, desprecio, vergüenza, miedo, alegría y el estado neutral.

Se utiliza el HOG y KNN porque arroja un valor de precisión de 71,42\%, sin embargo, con el HOG y SVM arroja un valor de precisión de $75 \%$ con la base KDEF. El algoritmo HOG con el KNN y con la base MUG arroja 65,71\%. 
Para la parte de reconocimiento facial se emplean varias etapas, la primera corresponde al preprocesamiento de las imágenes, para luego normalizarlas y eliminar los efectos de la luz (Figura 2). Luego, en una siguiente fase, se utiliza el procesamiento de extracción de características, que se emplea a través de un Histograma de Gradiente Orientado (HOG por sus siglas en inglés), que es el encargado de propiciar los datos de entrada para el entrenamiento y a su vez los datos de prueba para cada una de las emociones a clasificar. Más adelante en una tercera fase se configura un Support Vector Machine (SVM) como clasificador, junto con el algoritmo del vecino más cercano, k-Nearest Neighbours (KNN) que procesa cada dato nuevo evaluando los $\mathrm{k}$ vecinos cercanos, con el fin de determinar las distancias del nuevo elemento a cada uno de los ya existentes, de tal forma que hace posible la organización de las distancias en orden descendente y ubicar los elementos según el grupo al que corresponda. Este algoritmo no supervisado, se basa en varios atributos descriptivos y un solo atributo objetivo conocido como clase. Se evalúa además el desempeño del sistema considerando la técnica de la matriz confusión (CM). Esta matriz considera el número de predicciones de cada clase en cada una de sus columnas, y las instancias en la clase real están dadas por las filas de la matriz, así uno de los beneficios de las matrices de confusión es que facilitan la revisión del sistema y permiten determinar si este está confundiendo dos clases. El clasificador se evalúa tomando en cuenta las muestras de clases de la entrada, si estas producen muchos cambios en la tasa de error, entonces no serán representativas en cuanto la eficiencia del clasificador. El sistema es evaluado considerado las bases de datos JAFFE, KDE, WSEFEP y MUG, arrojando una tasa de predicción aproximada del 96,13\%.

Figura 2. Esquema de funcionamiento de reconocimiento de rostro para la detección de emociones

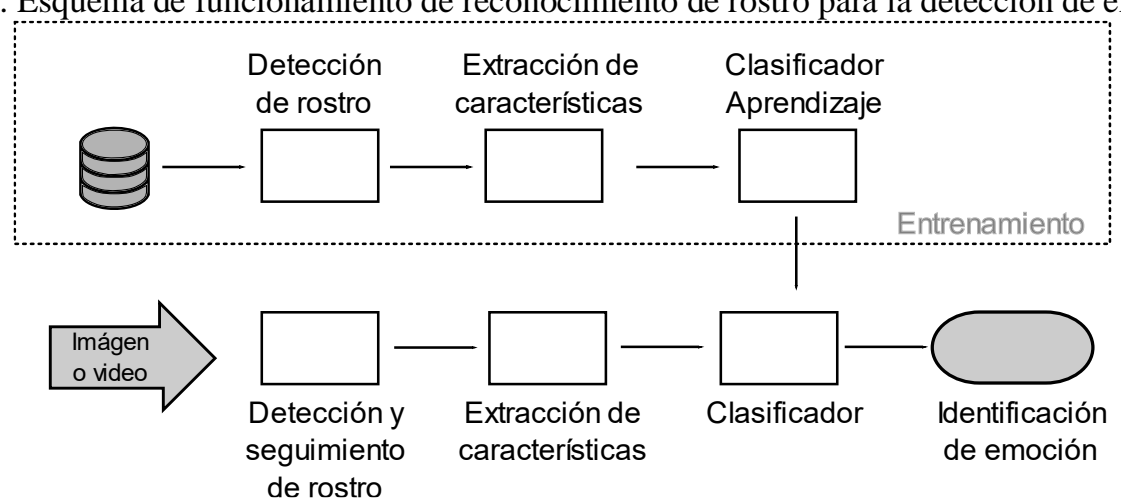

Ambos métodos fueron comparados para el proceso de determinación de emociones y valoración del estrés en las personas. Con ello fue posible obtener resultados apropiados que describen la relevancia de cada método. 


\section{RESULTADOS}

Los resultados obtenidos muestran que es posible determinar la emoción de una persona a partir de la fisionomía del rostro. Esta detección permite caracterizar los pequeños rastros inevitable que reflejan las emociones, producto de la actividad cerebral. El rostro permite reflejar las características más sublimes de las emociones, permitiendo que sea posible la detección de estas. Esta característica pudiese presentar algunas limitaciones, por ejemplo, los equipos de detección requieren una importante calidad técnica para la detección de las imágenes, iluminación de la habitación, disposición de los usuarios, distancia entre el usuario y el equipo, entre otras.

Fue posible reconocer la amplia variedad de estimadores de entre los cuales los más eficientes trabajan mediante redes neuronales, lo que le permite refinar la identificación de la emoción y por tanto su desempeño en el tiempo.

Además, fue posible verificar que la mejor combinación para el sistema es la utilización de los algoritmos HOG+SVM, con un $96.13 \%$ de precisión, lo cual presenta incertidumbres no verificadas dado que las emociones no son constantes en el tiempo, en un período corto de 10 segundos se ha observado cambios de hasta 3 emociones generadas posiblemente por recuerdos o asociaciones mentales del actor y considerando que las emociones no son constantes en todos los individuos.

Se descubrió que los estimadores son susceptibles de rasgos faciales referentes a la zona geográfica, etnia y características de la población. Además, un factor determinante en análisis de imagen es el entorno y condiciones de iluminación, deben ser estandarizados a fin de mejorar las estimaciones.

El análisis de las 7 emociones realizado, puede reducirse a las emociones más representativas para cuestiones de identificación del estrés, asi, el miedo y la sorpresa poseen ciertas similitudes, de igual manera con el enojo y el asco. Si se agrupan estas emociones, se pueden reducir hasta en un $30 \%$ los errores según Bartual (Bartual \& Herranz, 2017).

En múltiples ensayos realizados en la identificación de emoción, en base a la voz, se identifican como aspectos que afectan de forma relevante para una buena estimación a los parámetros como el sexo, edad y condición física y emocional.

Los ensayos realizados proporcionan un resultado aceptable para la identificación del estrés, sin embargo, este análisis debe refinarse con un sistema que mantenga la emoción por un tiempo prolongado a fin de mejorar esa predicción e incertidumbre. Una alternativa es el análisis en video, presentado en el trabajo de (Kulandai \& Sree, 2019), en él se pueden capturar de manera adecuada mucha más información, pero debe tomarse encuentra que según ensayos, la precisión se reduce en el mejor de los casos a un $87 \%$.

El clasificador analizado en este trabajo, es el tipo Adam, que es una combinación del Stochastic Gradient Descent with momentum y RMSprop (Kingma \& Ba, 2017). Este algoritmo de optimización 
promete una mayor eficiencia que otros algoritmos, haciendo que el error de la red llegue al mínimo en el menor tiempo posible, y posee similar precisión que métodos como:

- Convolutional neural network $(\mathrm{CNN})$ con un $97 \%$

- $\quad$ Patched based 3D Gabor con 94.48\% (Base C-K)

- SVM with clusterin algoritms, $94.34 \%$

La revision bibliográfica y el análisis del Sistema de detección de estrés resultaron un tema interesante para futuras investigaciones, ya que permitieron reconocer que las emociones humanas son complejas, son muy variadas y están sujetas a las características culturales, familiares y del entorno.

\section{CONCLUSIONES}

1. Las emociones son una característica humana que condiciona la conducta, y que puede afectar importantes aspectos de la vida.

2. Reconocer las emociones puede significar un hecho importante para el mejoramiento del individuo, que permita la valoración de sus actitudes y permita el control efectivo de las emociones que pueden perjudicar su vida social.

3. Las emociones pueden influir en la salud de las personas, reconocer los estados de estrés con antelación, pueden facilitar el mejoramiento de la salud y el uso eficiente de tratamientos medicos.

4. Un Sistema neuronal de reconocimiento de emociones puede facilitar la consulta médica preventiva. 


\section{REFERENCIAS}

Antón, E. (2013). Estrés laboral y variables biomédicas. Revista My Science Work.

Bartual, R., \& Herranz, J. (2017). Reconocimiento facial y reconocimiento anímico mediante análisis facial. Valencia: Universidad Politécnica de Valencia.

Bourdin, G. (2016). antropology of the emotions: cocepts and trends. Revista de Ciencias Antropológicas(67), 55-74.

Cárdenas., I., Ceballos., H., Shang-Hsueh., L., Pavez., W., \& Terrisse., C. (2010). Estudio acústico de la variación interlocutor en sujetos hablantes nativos del español de Santiago de Chile. . Chile: Tesis de Grado. Universidad de Chile.

Cercone, N., Yasmeen, F., \& Rahim, A. (2014). Detection of Stress by Voice \& Textual Information Analysis CSE6339 Project.

Fernández-Berrocal, P. (2009). Darwin y el misterio de las emociones. Málaga: UMA. SEDOC.

Giraldo., D., \& Quintero., O. (2010). Análisis de señales de audio utilizando la transformada de Gabor. $\begin{array}{lllll}\text { Recuperado el junio } & \text { de } & 2019, & \text { de }\end{array}$ https://repository.eafit.edu.co/.../29\%20Analisis_de_senales_audio_utilizando_transformada

Harlow, H., \& Harlow, M. (1962). Social Deprivation in Monkey. Scientific Americam(207), 136-146. Ibañez, C. (2009). Charles Darwin. Sociedad, 38-42.

Kingma, D., \& Ba, J. (2017). Adam: un método para la optimización estocástica. Cornell University, 9, $1-15$.

Kulandai, J., \& Sree, T. (2019). Reconocimiento de emociones faciales en videos usando HOG y LBP. (pág. 56). Bangalore, India: 4ta Conferencia internacional sobre tendencias recientes en electrónica, información, comunicación y tecnología (RTEICT). doi:10.1109 / RTEICT46194.2019.9016766

Martínez, E., \& Díaz, D. (2007). Una aproximación psicosocial al estrés escolar. Educación y Educadores, 10(2).

Montagu, A. (1959). Natural Selection and the Origin and Evolution of Weeping in Man. Science(130), 1572-1573.

Moure, P. (2011). De lo psicológico a lo fisiológico en la relación entre emociones y salud. Revista Psicología Científica, 13(19), 1-8.

Naranjo, M. (2009). Una revisión teórica sobre el estrpes y algunos aspectos relevantes de este en el ámbito educativo. Revista Educación, 33(2), 171-190.

Palacios, D. (2017). Contribución al estudio de selección de parámetros para identificación de estrés en la voz. España: Universidad Politécnica de Madrid.

Ramírez, E. (2001). Antropología "compleja" de las emociones humanas. Isegoría, 25, 177-200.

Reite, M., \& Short, R. (1981). Attachmente, loss and depression. Journal of Child Psychology and 
Psychiatr(2), 141-170.

Surrallés, A. (2005). Afectividad y epistemología de las ciencias huamanas. Revista de Antropología Iberoamericana, 1-15.

Wierzbicka, A. (1986). Human Emotions: Universal or Culture-Specific? . American Anthropologist, 88(3), 584-594. 